\title{
INTERPRETACIÓN DE LOS SISMOS PEQUEÑOS Y MODERADOS BAJO EL VOLCÁN IRAZÚ
}

\author{
INTERPRETATION OF SMALL TO MODERATE \\ SEISMICITY UNDER IRAZÚ VOLCANO
}

\author{
Monserrat Cascante-Matamoros ${ }^{1}$ \\ Hernán Porras-Espinoza ${ }^{2}$ \\ Universidad Nacional de Costa Rica, Costa Rica
}

\begin{abstract}
RESUMEN
El volcán Irazú históricamente ha presentado sismicidad volcano-tectónica esporádica. A partir de esta actividad sísmica hemos calculado un total de 7 mecanismos focales utilizando la polaridad de primeros arribos. Además, hemos realizado la inversión de esfuerzos utilizando el método PTB. Los resultados indican que la actividad sísmica se produce a profundidades entre 0-6 km en el flanco Oeste del macizo volcánico. Los mecanismos focales muestran una cinemática variada, los ángulos de los planos tienen una media de $55^{\circ}$ y la dirección de inclinación es hacia el Sur Oeste Oeste. La inversión de esfuerzos sugiere una extensión pura con un valor de $\mathrm{R}^{\prime}=0,5$. La profundidad donde ocurren los eventos sísmicos y el ángulo medio de los planos nos hace suponer que el límite dúctil-frágil se encuentra por encima de lo teóricamente establecido. Nosotros planteamos que la fuente sísmica bajo el volcán Irazú está directamente relacionada con la presencia de magma cerca de la superficie, el cual produce adelgazamiento de la corteza y fallamiento normal asociado.
\end{abstract}

Palabras clave: volcán Irazú, sismicidad, mecanismos focales, fallas normales.

1 Maestra (Universidad de Chile). Observatorio vulcanológico y sismológico de Costa Rica, Universidad Nacional de Costa Rica. Correo electrónico: monserrat.cascante.matamoros@una.cr

2 Maestro (Universidad de Chile). Observatorio vulcanológico y sismológico de Costa Rica, Universidad Nacional de Costa Rica. Correo electrónico: porrashernan@gmail.com 
Monserrat Cascante-Matamoros, Hernán Porras-Espinoza. Interpretation of small to moderate seismicity under Irazú Volcano

\begin{abstract}
Historically, Irazú volcano has experienced sporadic volcano-tectonic seismicity. Based on this seismic activity, we have calculated a total of 7 focal mechanisms using the polarity of first arrivals, and have calculated stress inversion using the PTB method. The results indicate that the seismic activity is between 0-6 km deep, mainly in the W flank of the volcanic massif. Focal mechanisms show a diverse cinematics, the angles of the planes have an average of $55^{\circ}$, and the direction of inclination is toward the SWW. Stress inversion suggests a pure extension with a value of $R^{\prime}=0,5$. Therefore, the depth of the seismic events and the average angle of the planes makes us suppose that the brittle-ductile transition is above the one theoretically established. We suggest that the seismic source under the Irazú Volcano is directly related to the presence of magma near the surface, which causes the thinning of the crust and the related normal faults.
\end{abstract}

Keywords: Irazú volcano, seismicity, focal mechanism, normal faults.

\title{
Introducción
}

El Irazú es un volcán escudo complejo de forma subcónica irregular, es el más alto de Costa Rica (3432 m.s.n.m) y se encuentra al NorEste de la ciudad de Cartago (Figura 1; Alvarado, 2011). Durante los últimos 50000 años el Irazú ha producido flujos de lava, erupciones de tipo estromboliano y freatomagmáticas, aunque no hay evidencia de erupciones plinianas en los últimos 11000 años (Alvarado y Schminke, 2013). Erupciones prehistóricas se han registrado en los años 640 a.C., 429 d.C., 686 d.C., 794 d.C., 1112 d.C. y 156 d.C. (Clark, et al., 2006) datadas por el método de radiocarbono $\left({ }^{\circ} \mathrm{C}\right)$. También existen registros históricos de actividad reciente e importante como la erupción reportada en 1723 y durante el siglo 20 (1917-1921, 1924, 1928, 1930, 1933, 1939-40 y 1963-65) (e.g. Alvarado, 1993, Alvarado et al., 2006). Las rocas del volcán Irazú son principalmente basaltos y andesitas que se superponen a ignimbritas dacíticas (Krushensky, 1972). 
Figura 1. Mapa de ubicación de la zona de estudio elaborado por los autores.

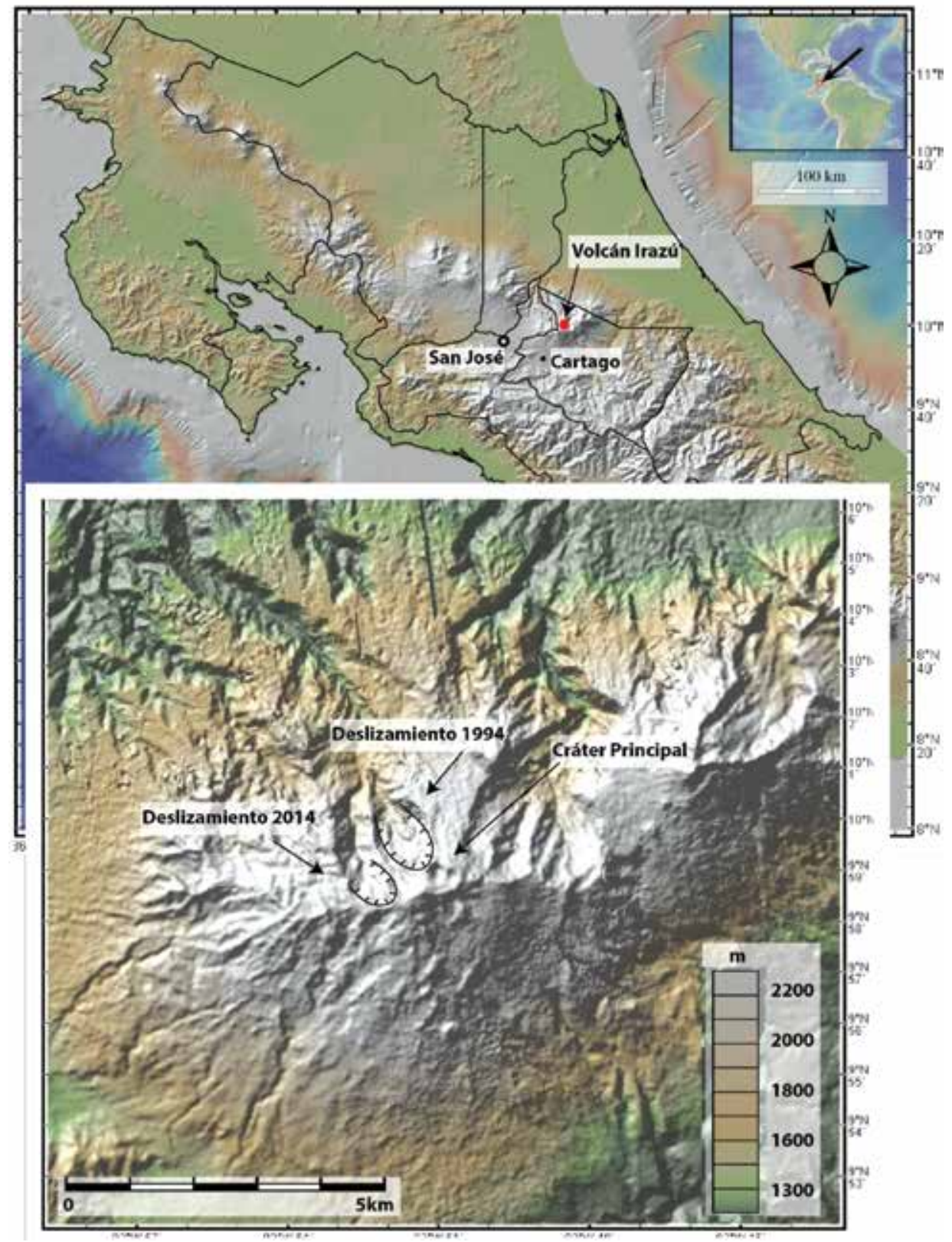

Fuente: Modelo de elevación digital GeoMapApp (Ryan et al., 2009). 
Al NorOeste del cráter principal, se encuentra un sector geológicamente inestable conocido como "las fumarolas" (Ulloa et al., 2013), la cual es una zona de intensa alteración hidrotermal, donde en diciembre de 1994 (Figura 1) se dio una supuesta explosión freática y un deslizamiento (Fernández et al., 1994; Barquero et al., 1995). El 17 de diciembre del 2014 se registró un deslizamiento al lado Oeste de la cima del volcán Irazú (Figura 1), el cual continúa activo (Pacheco et al., 2015).

Desde la última erupción a principios de 1960, el Volcán Irazú ha presentado sismicidad volcano-tectónica esporádica. El OVSICORI-UNA ha registrado este tipo de actividad desde 1984, con los inicios de la red sísmica. Alvarado y Schmincke (2013) con base en la actividad sísmica sugieren una fuente poco profunda $(<15 \mathrm{~km})$ con una intensidad máxima MM (escala Mercalli Modificada) de VI en la ciudad de Cartago y posible VII hacia el Volcán Irazú. Ellos sostienen que el mayor terremoto volcánico-tectónico podría haber tenido un $\mathrm{ML} \sim 4,8-5$.

Durante el periodo comprendido entre el 2006-2016 han ocurrido una serie de enjambres sísmicos, con magnitudes menores a los 4,1 ML, planteando la pregunta de si esta actividad se debe a fallamiento local o al ascenso del magma. En este trabajo, vamos a evaluar desde el punto de vista cinemático y dinámico los sismos relacionados al Volcán Irazú y discutir sobre la posible fuente sismogénica que los origina. Asimismo, comprender el mecanismo que genera la ruptura de la roca es importante para la interpretación de la dinámica interna y externa del macizo volcánico.

\section{Metodología}

Con fin de comparar cualitativa y cuantitativamente el campo de deformación inferido de las observaciones sísmicas, nosotros analizamos un total de 7 mecanismos focales elaborados a partir de la polaridad de los primeros arribos con un mínimo de 20 asociaciones, en el programa SEISAN. De este modo, se obtiene un conjunto final de mecanismos focales entre 2012 y 2015 con magnitudes locales (ML) que abarcan desde 1,9 hasta 4,1 (Tabla 1). 
Tabla 1. Sismos utilizados en la inversión de esfuerzos cinemáticos y dinámicos. latitud longitud profundidad magnitud dirección inclinación deslizamiento

\begin{tabular}{lllllllll}
\hline 1 & 18 julio 2015 & 9.965 & -83.868 & -1.9 & 3.2 & 143.5 & 55.2 & -83.9 \\
2 & 18 julio 2015 & 9.975 & -83.868 & -1.9 & 3.2 & 287.1 & 55.6 & 77.8 \\
3 & 25 julio 2015 & 9.976 & -83.87 & -3.6 & 2.9 & 176 & 48.4 & -48.1 \\
4 & 5 mayo 2012 & 9.963 & -83.857 & -4.1 & 2.8 & 233 & 28 & 43.2 \\
& & & & & & & & \\
5 & 18 julio 2015 & 9.966 & -83.874 & -5.1 & 3.2 & 9.1 & 42.3 & -52.9 \\
6 & 26 mayo 2015 & 9.961 & -83.889 & -5.3 & 4.1 & 71.2 & 85 & -8.7 \\
7 & 4 marzo 2012 & 9.967 & -83.854 & -8.8 & 1.9 & 73.7 & 26.8 & -67.3 \\
\hline
\end{tabular}

Fuente: catálogo sísmico OVSICORI-UNA (2017).

Para calcular el tensor de esfuerzos, nosotros asumimos que la dirección de deslizamiento sísmico ocurre en la dirección de máximo esfuerzo de cizalla (hipótesis de Wallace Bott, Bott, 1959). Utilizamos el método de inversión PBT, que determina el tensor de máxima presión, máxima tensión y el tensor intermedio (B) para la generación de un plano de falla (Zoback, 1992).

Este método de inversión es capaz de determinar los tres parámetros que reducen el tensor de esfuerzos $\left(\sigma_{1}, \sigma_{2}, \sigma_{3}\right)$ y la relación de aspecto (aspect ratio, $R$ ) donde; $R=\left(\sigma_{1}-\sigma_{2}\right) /\left(\sigma_{1}-\sigma_{3}\right)$ con $\sigma_{1} \geq \sigma_{2} \geq \sigma_{3}$ (Carey y Brunier, 1974; Angelier, 2002; Gephardt y Forsyth, 1984). El tipo de régimen de estrés puede ser expresado numéricamente usando un índice R' de 0 a 3 (Delvaux et al., 1997)

$\mathrm{R}^{\prime}=\mathrm{R}$ cuando $\sigma_{1}$ es vertical (régimen de estrés extensional).

$\mathrm{R}^{\prime}=2-\mathrm{R}$ cuando $\sigma_{2}$ es vertical (régimen de estrés desplazamiento de rumbo).

$\mathrm{R}^{\prime}=2+\mathrm{R}$ cuando $\sigma_{3}$ es vertical (régimen de estrés compresional).

\section{Resultados}

Desde el año 2006 se han registrado un total de 320 eventos sísmicos bajo el Volcán Irazú. Estos eventos se concentran principalmente en el flanco Oeste (Figura 2) y se dispersan hacia el flanco Este del volcán. En profundidad estos sismos se ubican entre los 0-6 km y tienden a formar un 
alineamiento con inclinación hacia el Oeste. A partir de la distribución de sismos (figura 2), se infiere que el patrón deformación no es uniforme en todo el Volcán Irazú.

Figura 2. Perfil topográfico del Volcán Irazú. La figura muestra la sismicidad registrada desde el año 2006 bajo este volcán. Los sismos en rojo representan los eventos con mecanismo focal calculado. La numeración representa el ID en la tabla 1.

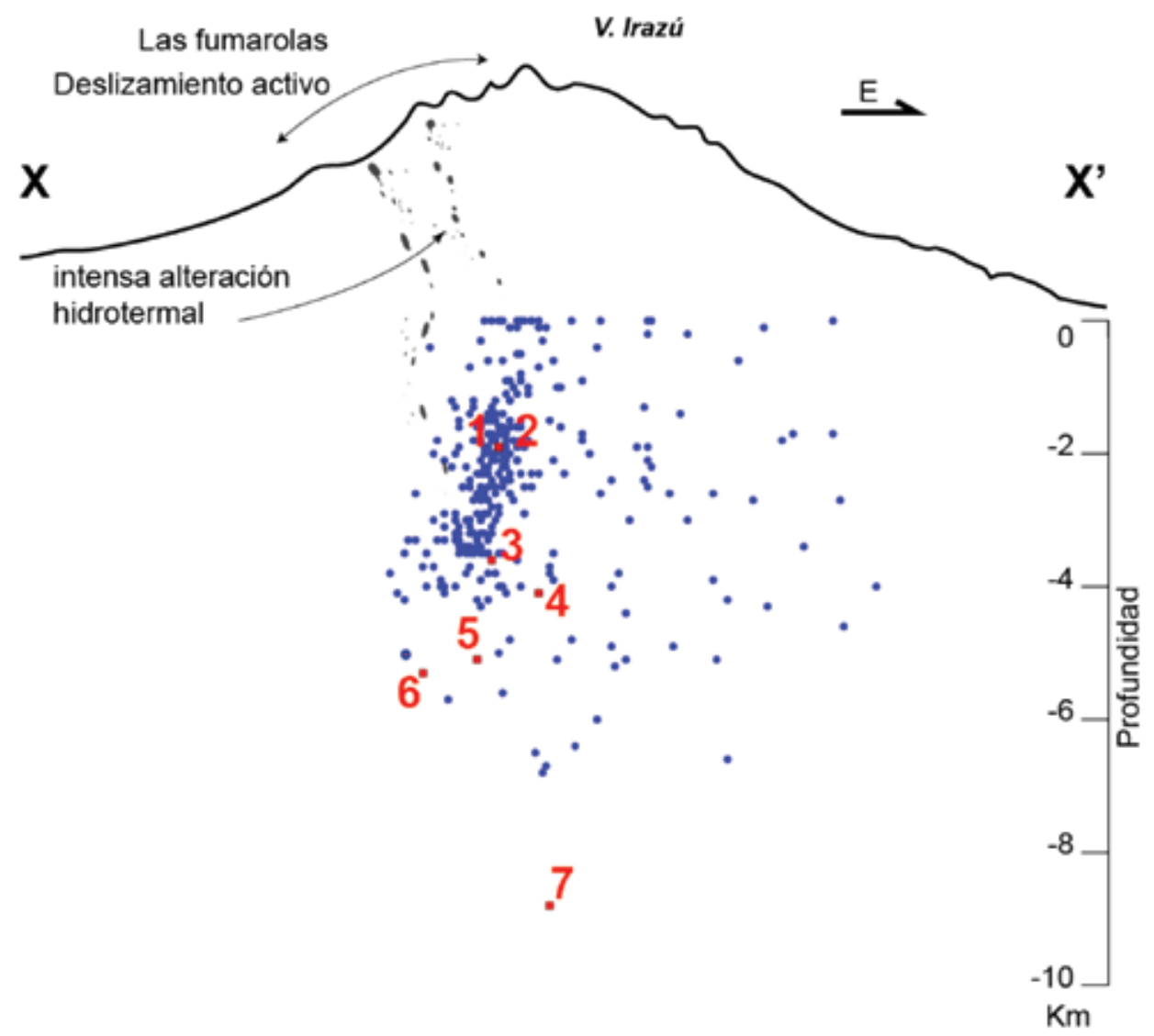

Fuente: Elaborado por los autores (2017).

La cinemática observada en los mecanismos focales se caracteriza por un fallamiento normal y poco deslizamiento en la componente de 
rumbo. Se han obtenido además dos eventos con una marcada componente inversa a profundidades de 1,9 y 4,1 km (Figura 3).

Figura 3. Mecanismos focales calculados en el Volcán Irazú. La numeración representa el ID de la tabla 1. x-x' es la línea e perfil en la figura 2

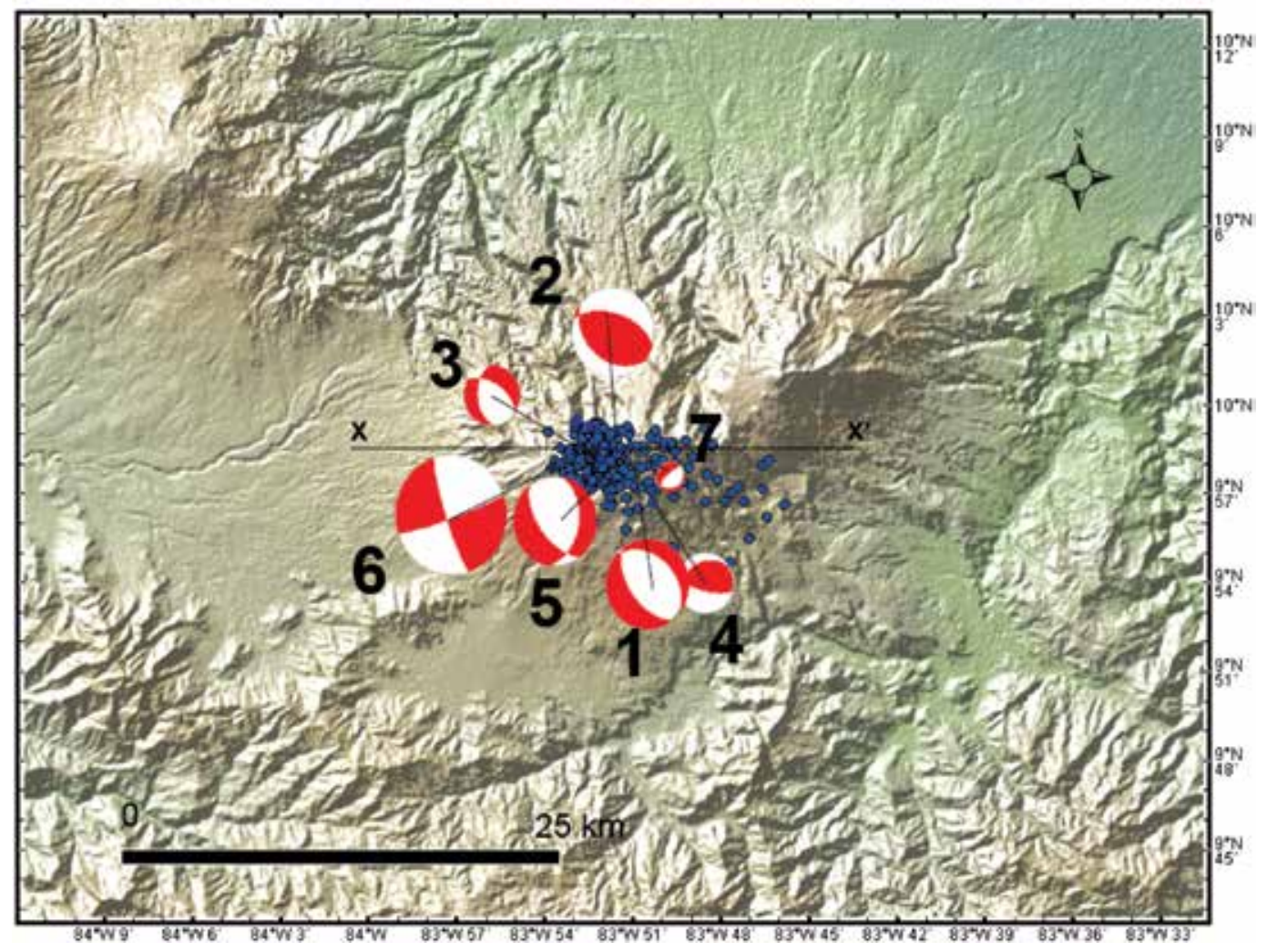

Fuente: Modelo de elevación digital: GeoMapApp (Ryan et al., 2009); catalogo sísmico: OVSICORI-UNA.

La dirección de rumbo predominante para todos los eventos analizados es NorOeste-SurEste con un azimut cercano al NorNorEste y un ángulo de inclinación de $60^{\circ}$ (Figura 4). Los ángulos de inclinación de los planos de falla son moderados, entre los $60^{\circ}$ y $30^{\circ}$ con una media en $55^{\circ}$. Se observan dos tendencias muy cercanas entre sí para el esfuerzo horizontal máximo $\left(\mathrm{SH}_{\max }\right)$ en dirección NorEsteEste-SurOesteOeste, perpendicular a la dirección de rumbo y paralelo a la inclinación (Figura 4). 
Monserrat Cascante-Matamoros, Hernán Porras-Espinoza. Interpretation of small to moderate seismicity under Irazú Volcano
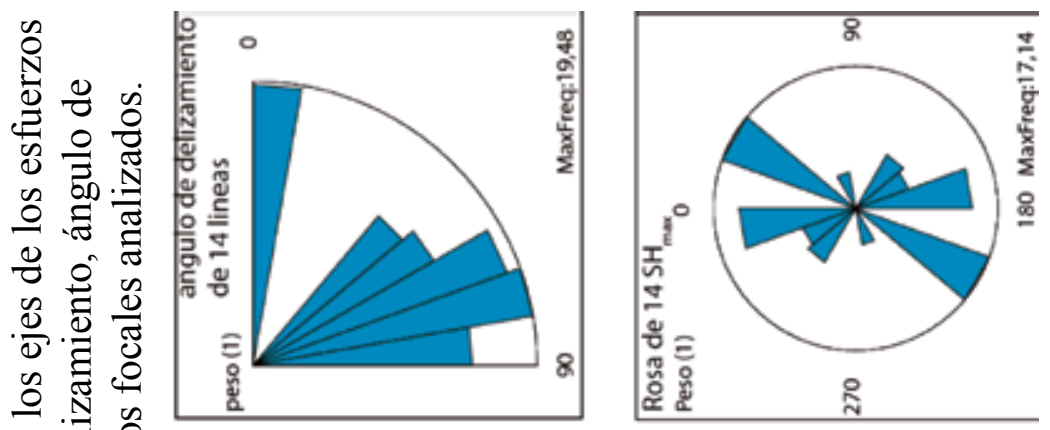

过

:웡

:
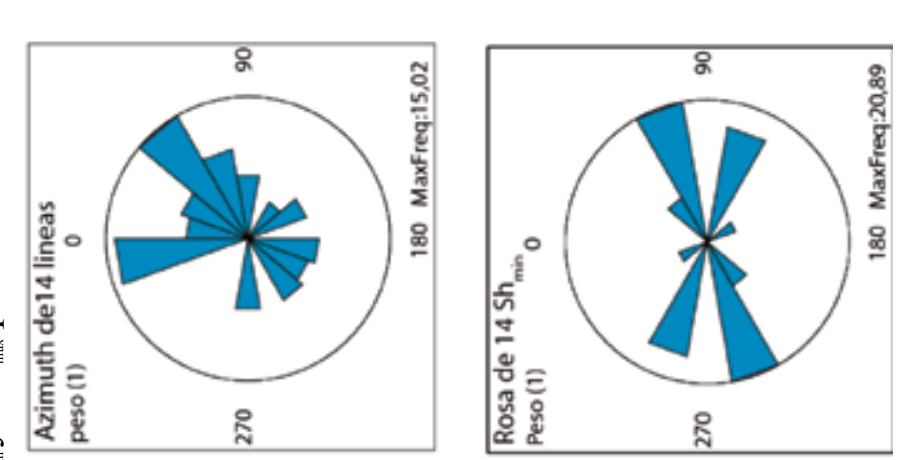

$\overrightarrow{0}>$

ค

.
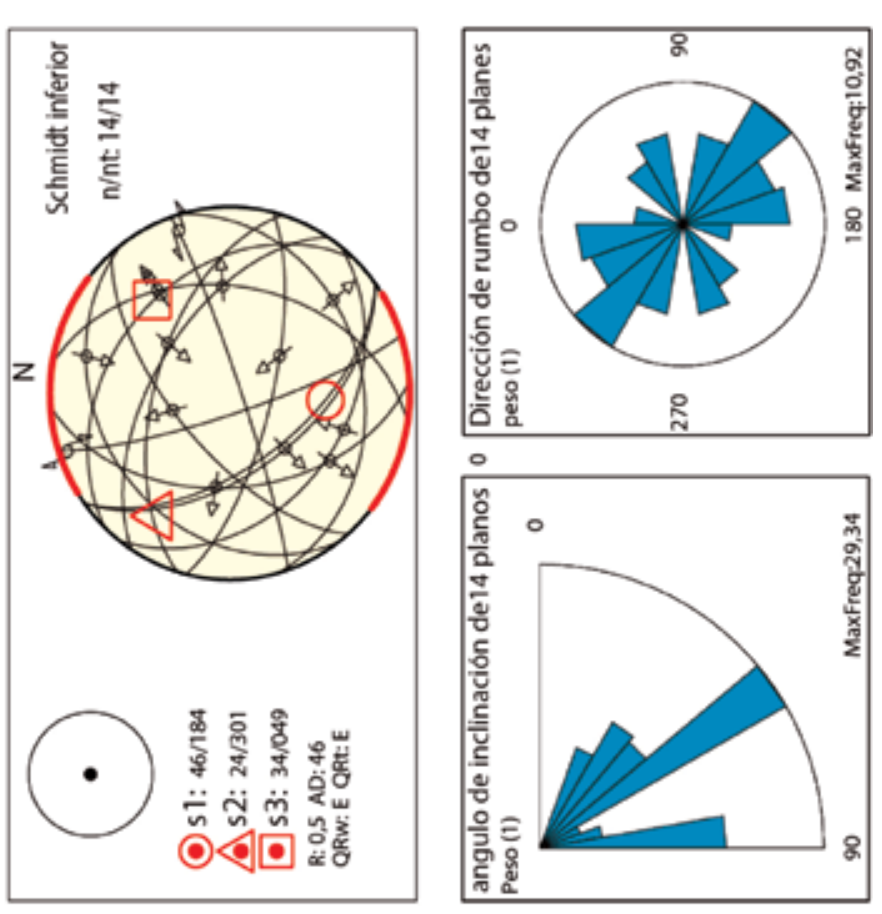

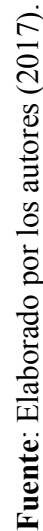
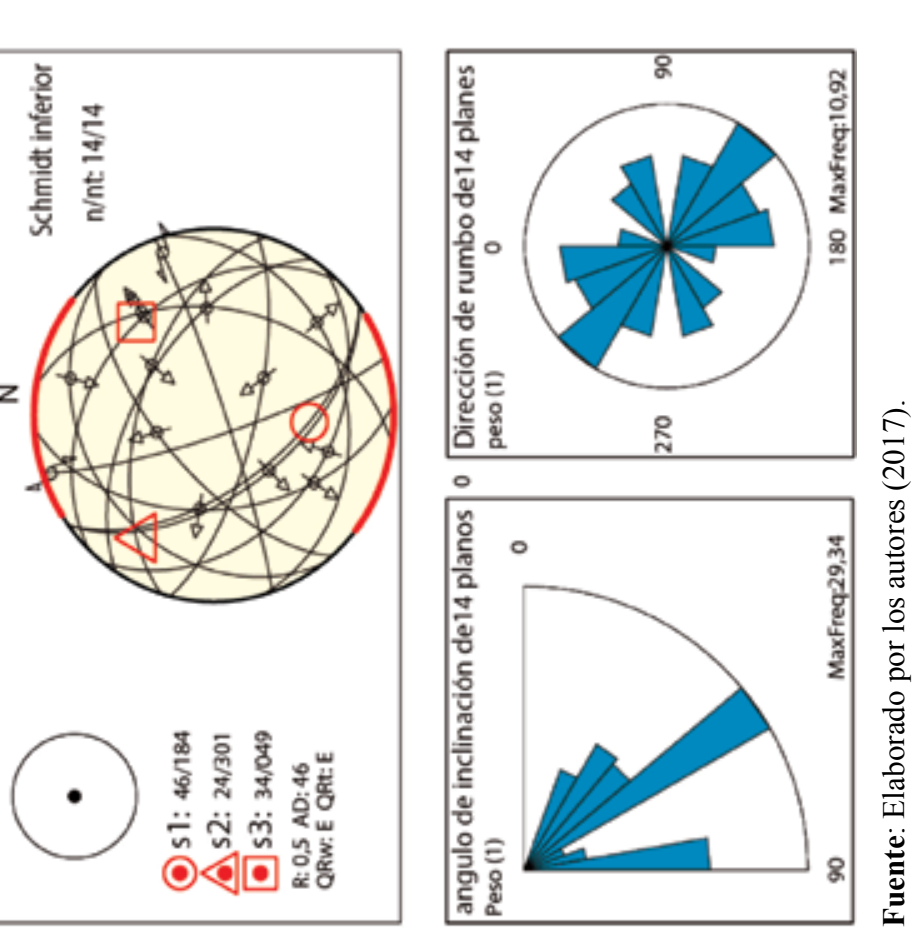
Los resultados de la inversión de estrés revelan que las orientaciones de los ejes principales son sub-vertical para $\sigma_{1}$ y cerca de la horizontal para $\sigma_{2}$ y $\sigma_{3}$. La solución obtenida muestra un sistema de extensión radial en dirección NorEste-SurOeste. El eje $\sigma_{2}$ corresponde al esfuerzo $\mathrm{SH}_{\max }$ en dirección $28^{\circ} / 304^{\circ}$ con un valor de $\mathrm{R}^{\prime}=0,5$ (Figura 4).

\section{Discusión}

Los sismos bajo un edificio volcánico pueden reflejar ya sea, la fracturación hidráulica (si la presión del fluido supera el esfuerzo principal) o deslizamiento sobre las fracturas preexistentes desencadenadas por la reducción de la tensión normal efectiva resultante de un aumento de la presión del fluido (Shelly y Hill, 2011). Esta presión de fluidos estaría asociado con las variaciones en la permeabilidad proporcionada por fracturas en la parte superior del Volcán Irazú. Una consecuencia de esta migración de fluidos se puede observar al NorOeste del Volcán Irazú, en las "fumarolas" (Ulloa et al., 2013, Figura 2), donde se han presentado zonas de deslizamientos y alteración hidrotermal importante, lo cual ha generado una perturbación del sistema cercano al volcán. Nuestros datos muestran que la dirección del eje de esfuerzos horizontal mínimo $\left(\mathrm{Sh}_{\min }=\sigma_{3}\right.$ para sistemas extensionales), donde se da la extensión, es en dirección NorEsteEsteSurOesteOeste. Justo en el sector Oeste, conocido como las "torres" se ha reactivado un deslizamiento desde el 2014 (Pacheco et al., 2015) que ha afectado la dinámica externa del volcán (Figura 2). Este deslizamiento puede atribuirse a la dilatación positiva generada en un sistema extensivo (Allmendinger et al., 2007).

Por otro lado, el análisis cinemático muestra los tres tipos posibles de movimientos dentro del mismo enjambre sísmico. Los ejes de esfuerzos principales $\left(\sigma_{1}, \sigma_{2}, \sigma_{3}\right)$ mantienen magnitudes relativas muy cercanas entre sí. Esta simetría entre los esfuerzos es bien representada en el valor R' de 0.5 . Esto sugiere que la zona se encuentra bajo una fuerte presión de fluidos, lo cual facilita la permutación de esfuerzos locales (Figura 4).

La inversión de esfuerzos revela un sistema de extensión pura, el cual se caracteriza por un régimen de fallamiento normal y se define por un máximo esfuerzo de compresión principal en la vertical (Anderson, 1951). Las fallas normales activas comúnmente tienen altas inclinaciones cerca de la superficie y son cada vez más suaves en profundidad conforme 
se acercan al límite frágil-dúctil, produciendo una geometría lístrica (Yamada y McClay, 2003). Las inclinaciones entre $70^{\circ}-90^{\circ}$ son comunes en la corteza superior quebradiza (aprox. 0-2 km) en una variedad de rocas incluyendo tobas (Day et al., 1998), basaltos (Opheim y Gudmundsson, 1989; Angelier et al., 1997; Dauteuil et al., 2001), calizas (Ferrill y Morris, 2003; Ferrill et al., 2004), y las rocas sedimentarias clásticas (Walsh y Watterson, 1988). Las inmersiones de $60^{\circ}$ son comunes a una profundidad de $2-5 \mathrm{~km}$, e inclinaciones de $35^{\circ}-55^{\circ}$ son comunes en la parte inferior de la corteza quebradiza (aprox. $>5 \mathrm{~km}$; Jackson y White, 1989; Collettini y Sibson, 2001).

Nuestros mecanismos focales muestran los posibles planos de falla con inclinaciones entre $35^{\circ}-55^{\circ}$, lo cual sugiere que se encuentran en la parte inferior de la corteza quebradiza. Por otro lado, los sismos desde los cuales obtuvimos los mecanismos focales se encuentran en su mayoría entre los 0 y 5,3 km de profundidad (Tabla 1). Esto nos hace suponer que el límite frágil-dúctil se encuentra por encima de lo teóricamente establecido.

La profundidad del límite frágil-dúctil bajo un edificio volcánico puede estar controlado por la presencia de un cuerpo magmático (Buck et al., 2006). La influencia de un cuerpo magmático adelgaza la litósfera, la cual se vuelve más caliente y por lo tanto más débil (Kusznir y Park, 1984). A medida que progresa el afinamiento de la litósfera y de la corteza por ascenso del magma, la zona de transición frágil-dúctil se torna somera (Kusznir y Park, 1987; Reston y Pérez-Gussinyé, 2007). Esta variación en la profundidad del límite frágil-dúctil favorece la deformación frágil en vez de deformación plástica o reptaje (creep) de las rocas generando extensión. Los procesos por los cuales se acomoda la extensión generada son básicamente dos: fallamiento normal y magmatismo (Hayward y Ebinger 1996; Wright et al. 2006; Rowland et al. 2007).

Por lo tanto, la sismicidad registrada bajo el Volcán Irazú es originada por la presencia de un cuerpo magmático somero que adelgaza la corteza y la torna quebradiza, facilitando así el fallamiento normal. Una consecuencia externa directa de la actividad sísmica del volcán son la fumarolas y los deslizamientos ubicados en el sector Oeste, los cuales se originan en un sector dominado por un régimen extensivo. 


\section{Conclusiones}

Los mecanismos focales de los sismos que han tenido lugar en el Volcán Irazú se han determinado mediante el análisis de las polaridades de las ondas $\mathrm{P}$ de sismogramas regionales. Los principales resultados de un conjunto de datos de 7 soluciones focales fiables son:

1. Los sismos se caracterizan por un mecanismo de fallamiento normal e inverso con moderados ángulos de inmersión $\left(35^{\circ}-55^{\circ}\right)$ y pequeños componentes de rumbo. Las profundidades de todos los terremotos analizados se encuentran en el rango de 1-6 km.

2. Los resultados del análisis cinemático revelan una tendencia general de extensión NorEsteEste-SurOesteOeste para el flanco Oeste del Volcán Irazú.

3. La inversión dinámica a partir de los datos de mecanismos focales muestran que el campo de esfuerzos actual se caracteriza por un régimen extensional $\left(\mathrm{R}^{\prime}=0,5\right)$ y una dirección horizontal de extensión $\left(\mathrm{Sh}_{\text {min }}\right)$ cercana al NorEsteEste-SurOesteOeste. Las orientaciones de los ejes principales de esfuerzos son $\sigma_{1}$ vertical y horizontal para $\sigma_{2}$ y $\sigma_{3}$.

4. La fuente sismogénica bajo el Volcán Irazú está asociada a la presencia de un cuerpo magmático cercano a la superficie que produce una somerización del límite frágil-dúctil generando una corteza adelgazada y quebradiza que favorece el fallamiento normal.

\section{Referencias}

Allmendinger, R., Reilinger, R. y Loveless, J. (2007). Strain and Rotation Rate from GPS in Tibet, Anatolia, and the Altiplano. Tectonics, 26(TC3013), 1-18. doi:10.1029/2006TC002030.

Alvarado, G. E. (1993). Volcanology and Petrology of Irazú Volcano, Costa Rica. Kiel: Univ. Kiel.

Alvarado, G. E. (2011). Los volcanes de Costa Rica: Geología, historia, riqueza natural y su gente. San José: EUNED.

Alvarado, G. E., y Schmincke, H. U. (2013). The 1723 A.D. Violent Strombolian and Phreatomagmatic Eruption at Irazú Volcano, Costa Rica. Revista Geológica de América Central, 48, 41-61.

Alvarado, G. E., Carr, M., Turrin, B., Swisher, C., Schmincke, H., y Hudnut, K. (2006). Recent volcanic history of Irazú volcano, Costa Rica: Alternation 
and mixing of two magma batches, implying at least two intracrustal chambers. En Rose, W., Bluth, G., Carr, M., Ewert, J., Patino, L., y Vallance, J. Natural Hazards in Central America, 412, pp. 259-276. Geological Society of America Special Paper. doi:10.1130/2006.2412(14).

Anderson, E. M. (1951). The Dynamics of Faulting, Etc. (Revised.). Edinburgh, London.

Angelier, J. (2002). Inversion of earthquake focal mechanisms to obtain the seismotectonic stress IV - a new method free of choice among the nodal planes. Geophysical Journal International, 150, 588-609.

Angelier, J., Bergerat, F., Dauteuil, O. y Villemin, T. (1997). Effective tension-shear relationships in extensional fissure swarms, axial rift zone of northeastern Iceland. Journal of Structural Geology, 19, 673685. doi:10.1016/S0191-8141(96)00106-X.

Barquero, R., Mora, M., Madrigal, L., Vargas, I., Arias, F. y Soto, G. (1995). Resumen anual de sismos y actividad volcánica en Costa Rica durante 1994. OVSICORI [Informe Interno].

Bott, M. P. (1959). The mechanics of oblique slip faulting. Geological Magazine, 96, 109-117.

Buck, R. W., Einarsson, P. y Brandsdóttir, B. (2006). Tectonic stress and magma chamber size as controls on dike propagation: Constraints from the 1975-1984 Krafla rifting episode. Journal of Geophysical Research, 111(B12404), 1-15. doi:10.1029/2005JB003879.

Carey, E. y Brunier, B. (1974). Analyse théorique d'un modèle mécanique élémentaire aplié à l'étude d'une population de failles. Comptes Rendus de l'Académie des Sciences Paris, 279, 891-894.

Clark, S., Reagan, M., y Trimble, D. (2006). Tephra deposits for the past 2600 years from Irazú volcano, Costa Rica. En Rose, W., Bluth, G., Carr, M., Ewert, J., Patino, L., y Vallance, J. Natural Hazards in Central America, 412, pp. 225-234 Geological Society of America Special Paper. doi:10.1130/2006.2412(12).

Collettini, C. y Sibson, R. H. (2001). Normal faults, normal friction? Geology, 29, 927-930. doi:10.1130/0091-7613(2001)0292.0.CO;2.

Dauteuil, O., Angelier, J., Bergerat, F., Verrier, S. y Villemin, T. (2001). Deformation partitioning inside a fissure swarm of the northern Iceland rift. Journal of Structural Geology, 23, 1359-1372. doi:10.1016/ S0191-8141(01)00002-5. 
Day, W. C., Dickerson, R. P., Potter, C. J., Sweetkind, D. S., San Juan, C. A., Drake, R. M. y Fridrich, C. J. (1998). Bedrock geologic map of the Yucca Mountain area, Nye County, Nevada. [Mapa]. Escala 1:24000. U.S. Geological Survey Miscellaneo Investigations Series Map. Nevada, EE.UU. I-2627.

Delvaux, D., Moeys, R., Stapel, G., Petit, C., Levi, K., Miroshnichenko, A., Ruzhich, V. y San'kov, V. (1997). Paleostress reconstructions and geodynamics of the Baikal region, central Asia, Part 2. Cenozoic rifting. Tectonophysics, 282(1), 1-38.

Fernández, E., Barquero, J., Barboza, V., Van Der Laat, R., Malavassi, E., Sáenz, R., Marino, T. y Martínez, M. (1994). Estado de los volcanes. Boletín de Vulcanología. OVSICORI-UNA [Informe Interno].

Ferrill, D.A.y Morris,A.P.(2003). Dilational normal faults. Journal of Structural Geology, 25, 183-196. doi:10.1016/S0191-8141(02)00029-9.

Ferrill, D. A., Sims, D. W., Waiting, D. J., Morris, A. P., Franklin, N. y Schultz, A. L. (2004). Structural framework of the Edwards Aquifer recharge zone in south-central Texas. Geological Society of America Bulletin, 116, 407-418. doi:10.1130/B25174.1.

Gephart, J. W. y Forsyth, D. W. (1984). An improved method for determining the regional stress tensor using earthquake focal mechanism data: Application to the San Fernando earthquake sequence. Journal of Geophysical Research, 89, 9305-9320.

Hayward, N. y Ebinger, C. (1996). Variations in the along-axis segmentation of the Afar rift system. Tectonics, 15, 244-257.

Jackson, J. A. y White, N. J. (1989). Normal faulting in the upper continental crust: Observations from regions of active extension. Journal of Structural Geology, 11, 15-36. doi:10.1016/0191-8141(89)90033-3. Krushensky, R. D. (1972). Geology of Istarú Quadrangle. Washington D.C.: U.S. Geological Survey Bulletin, 1358.

Kusznir, N. J., y Park, R. G. (1984). Intraplate lithosphere deformation and the strength of the lithosphere. Geophysical Journal International, $79(2), 513-538$.

Opheim, J. A. y Gudmundsson, A. (1989). Formation and geometry of fractures, and related volcanism, of the Krafla fissure swarm, northeast Iceland. Geological Society of America Bulletin, 101, 16081622. doi:10.1130/0016-7606(1989)1012.3.CO;2. 
Pacheco, J. P., Muller, C., Avard, G., Cascante, M. y Martínez, M. (2015). Estado de los volcanes. Boletín de Vulcanología. OVSICORI [Informe Interno].

Reston, T. J. y Pérez-Gussinyé, M. (2007). Lithospheric extension from rifting to continental breakup at magma-poor margins: rheology, serpentinisation and symmetry. International Journal of Earth Sciences, 96, 1033-1046.

Rowland, J., Baker, E., Ebinger, C., Keir, D., Kidane, T., Biggs, J., Hayward, D. y Wright, T. (2007). Fault growth at a nascent slow-spreading ridge: 2005 Dabbahu rifting episode, Afar. Geophysical Journal International, 171, 1226-1246.

Ryan, W. F., Carbotte, S. M., Coplan, J. O., O’Hara, S., Melkonian, A., Arko, R., Weissel, R.A., Ferrini, V., Goodwillie, A., Nitsche, F., Bonczkowski, J. y Zemsky, R. (2009). Global Multi-Resolution Topography synthesis. Geochemistry Geophysics Geosystems, 10(Q03014), 1-9. doi:10.1029/2008GC002332.

Shelly, D. R. y Hill, D. P. (2011). Migrating swarms of brittle-failure earthquakes in the lower crust beneath Mammoth Mountain, California. Geophysical Research Letters, 38(L20307), 1-6. doi:10.1029/2011GL049336.

Ulloa, A., Campos-Fernández, C. S. y Rojas, L. (2013). Cueva los minerales, volcán Irazú, Costa Rica: descripción, mineralogía y origen. Revista Geológica de América Central, 48, 169-187.

Walsh, J. J. y Watterson, J. (1988). Dips of normal faults in British Coal Measures and other sedimentary sequences. Journal of the Geological Society, London, 145, 859-873.

Wright, T., Ebinger, C., Biggs, J., Ayele, A., Yirgu, G., Keir, D. y Stork, A. (2006). Magma-maintained rift segmentation at continental rupture in the 2005 Afar dyking episode. Nature, 422, 291-294.

Yamada, Y. y McClay, K. (2003). Application of geometric models to inverted listric fault systems in sandbox experiments. Paper 1: $2 \mathrm{D}$ hanging wall deformation and section restoration. Journal of Structural Geology, 25(9), 1551-1560. doi:10.1016/S0191-8141(02)00181-5.

Zoback, M. L. (1992). First- and second-order patterns of stress in the lithosphere: The World Stress Map Project. Journal of Geophysical Research, 97(B8), 11703-11728. doi:10.1029/92JB00132. 